\title{
International Journal of Anesthesia and Relaxation
}

\section{Obstetric Critical Care: Overview of Relevant Issues}

Bhiwal Kumar Anil ${ }^{1}$, Gupta Sunanda ${ }^{* 2}$

${ }^{1}$ Assistant Professor Department of Anaesthesiology, Geetanjali Medical College and Hospital, Udaipur, Rajasthan,India

${ }^{2}$ Professor and Head, Department of Anaesthesiology, Geetanjali Medical College and Hospital, Udaipur, Rajasthan,India

Corresponding Author: Gupta Sunanda, Professor and Head Department of Anaesthesiology, Geetanjali Medical College and Hospital, Udaipur, Rajasthan, India. Email: sunandagupta@hotmail.com

Citation: Gupta Sunanda et al. (2017), Obstetric Critical Care: Overview of Relevant Issues. Int J Ane \& Rel. 1:2, 19-22.

DOI: $10.25141 / 2575-9736-2017-2.0019$

Copyright: C2017 Gupta Sunanda et al. This is an open-access article distributed under the terms of the Creative Commons Attribution License, which permits unrestricted use, distribution, and reproduction in any medium, provided the original author and source are credited

Received: October 11, 2017; Accepted: November 06, 2017; Published: November 30, 2017

Abstract:

Critically ill obstetric patients present a unique challenge to the obstetrician, anaesthesiologist and intensivists. Maternal mortality has shown a sharp decline in the developed countries but in developing countries it is still high due to lack of good maternal antenatal services and obstetric intensive care. The indications for admission of a parturient to an ICU are obstetric complications as well as non obstetric indications. Optimal management of the critically ill obstetric patient involves early detection and multi disciplinary approach with close collaboration of the critical care team including obstetricians, midwives, anesthesiologists and neonatologists based on knowledge of physiological and pharmacological alterations. This article aims to provide a comprehensive review for the management of critically ill obstetric patient including monitoring, ventilator strategies, drugs, nutrition, thromboprophylaxis, prognostic indicators and recent update on commonly encountered situations

Keywords: Critical Illness, Obstetric, ICU

\section{Introduction:}

Critical illness in the parturient presents a unique challenge to the obstetrician, anaesthesiologist and intensivists. Complications can occur any time during pregnancy and puerperium which may progress to severe maternal morbidity also known as 'near-miss' maternal mortality, thus necessitating critical care support. The medical management of the critically ill obstetric patient requires a complete knowledge of the altered physiology of pregnancy as well as the superimposed acute pathological insult, demanding management by a multidisciplinary team including obstetrician, intensivists and anesthesiologists.

The incidence of ICU admission of an obstetric patient in developed countries is 2-4/1000 deliveries, while in developing countries the incidence is $2-13.5 / 1000$ deliveries. ${ }^{[1]}$ Maternal mortality has shown a sharp decline in the developed countries but in developing countries it is still high due to lack of good maternal antenatal services and obstetric intensive care. The anaesthesiologist plays a key role in the management of high risk pregnancies, as they are trained in advanced life support and resuscitation and they should educate the medical and paramedical staff involved in the labour ward for the early recognition, monitoring and treatment of such patients. ${ }^{[2]}$

Indications for ICU admission:

The reasons for obstetric admissions to an ICU include obstetric
$(47 \%-93 \%)$ as well as non obstetric indications. ${ }^{[3]}$

Obstetric indications: Antepartum and postpartum haemorrhage, hypertensive disease like preeclampsia and eclampsia, puerperal sepsis are the most frequent indications.

Non obstetric indication: maternal cardiac disease, trauma, anaesthetic complications, cerebrovascular accidents, malaria, viral hepatitis, cerebral venous thrombosis, poisoning and drug overdose.

Presence of other comorbidities like diabetes and renal diseases also contribute negatively to maternal outcome in the ICU, and hence simultaneous stabilization of mother and fetus should be our goal. ${ }^{[4]}$

Altered Physiology in Pregnancy Relevant to Criticality:

Almost all organ systems are affected by the physiological changes that occur during pregnancy; however cardiovascular and the respiratory systems are the most important systems that affect criticality in the obstetric patient.

Cardiovascular system: The blood volume is increased by $50 \%$ during pregnancy but plasma volume increases much more as compared to the corpuscular volume which causes physiological anaemia. The Heart rate and cardiac output increases while Systemic and Pulmonary vascular resistance is decreased. In Preeclampsia and Eclampsia, patients have relative haemoconcen- 
tration and thus are much more susceptible to blood loss during delivery. These patients are more prone to pulmonary edema due to increased vascular permeability and decreased colloid osmotic pressure. After 20 weeks gestation they are prone to supine hypotension syndrome due to the pressure of the gravid uterus on the descending aorta and inferior vena cava which is relieved in the lateral position or on displacement of the uterus.

Respiratory system: Minute volume is increased due to increased tidal volume and respiratory rate leading to respiratory alkalosis with decrease in serum bicarbonate levels to compensate this alkalosis. During pregnancy Residual volume, functional residual capacity and total lung capacity are decreased so pregnant patients are more prone to hypoxia.

Hematological changes: Total leukocyte count may increase upto $25000 / \mathrm{mm} 3$ which may lead to misdiagnosis of infection in ICU. Platelet counts may decrease but coagulation process is deranged with increased levels of almost all procoagulants and decrease in fibrinolysis leading to increased incidence of DVT and pulmonary embolism.

Urinary system: Renal plasma flow and GFR are increased while serum creatinine is decreased from 1.2 to $0.8 \mathrm{mg} / \mathrm{dl}$.

Specific Obstetric Emergencies:

Obstetric haemorrhage: Massive obstetric haemorrhage is defined as blood loss $>1500 \mathrm{ml}$, a decrease in $\mathrm{Hb}$ of $<4 \mathrm{~g} / \mathrm{dl}$. It is responsible for more than $30 \%$ of all maternal deaths in low income countries and over $10 \%$ of maternal deaths in high income countries. The management involves treatment of uterine atony (uterine massage, oxytocin, and prostaglandin analogues), surgical intervention (uterine tamponade or compression packing, balloon compression sutures), arterial ligation and selective radiological embolization and even hysterectomy. The goal directed resuscitation strategy using point of care coagulation tests (thromboelastography and rotational thromboelastometry) is considered important for optimal management. ${ }^{[5]}$ Use of PRBC, FFP and platelets according to massive transfusion protocols with early use of fibrinogen concentrates and tranexamic acid is life saving.

Preeclampsia: The complications of severe preeclampsia include HELLP syndrome, pulmonary edema, renal failure, Cerebrovascular accidents and Eclampsia which results in ICU admission. Management includes control of hypertension and seizure, restricted fluid administration to avoid pulmonary edema and early delivery of the fetus.

Sepsis: Maternal sepsis causes $15 \%$ of maternal death worldwide. Puerperal sepsis and UTI are the most important causes of sepsis in developed nations but Malaria, HIV and community acquired pneumonia are important causes in developing countries. Adequate volume expansion to optimize cardiac output, antibiotic regimens to treat gram negative, gram positive and anaerobic infections along with invasive haemodynamic monitoring to guide inotropic therapy is indicated as per the recent guidelines of surviving sepsis compaign. ${ }^{[6]}$

Amniotic fluid embolism (AFE): AFE is a clinical diagnosis of exclusion and has a high mortality rate. The biomarkers used for diagnosis include Zinc corproporphyrin-1, Sialyl Tn antigen (STN), Complement C-3, C-4, interlukin-8, insulin- like growth factor-binding protein-1. The management of AFE is mainly supportive and should be managed in an ICU by a multidisciplinary team.

Heart disease: Cardiac disorders affect 1-3\% pregnant women and account for $10-15 \%$ Maternal Mortality. Peripartum cardiac disease in pregnancy range from congenital anomalies to valvular heart disease, myocardial infarction, dilated cardiomyopathy and pulmonary hypertension which is further worsened by the high cardiac outputduring pregnancy. The management strategy of shock in such cases includes volume replenishment, appropriate antibiotics, vasopressors, early revascularization, inotropic support and even mechanical circulatory support for refractory cases. ${ }^{[7]}$ Severe Mitral stenosis may present with pulmonary edema and atrial fibrillation while Aortic stenosismay develop fatal arrhythmia and refractory heart failure. AHA recommends beta blockers, nondihydropyridine, calcium channel blockers and digoxin for $\mathrm{AF}$ in pregnancy. Uteronic drugs should be used cautiously at the lowest effective dose and never as a bolus. Echocardiography should be used as a point of care in these patients.

Cardiopulmonary arrest: The patient positioning and role of perimortem caesarean delivery are two main problemsduring CPR in pregnant patient. Left lateral tilt decreaseschest compression quality hencemanual uterine displacement by another care provider can relieve aortocaval compression.If, gestational age is 20 weeks then perimortem caesarean delivery should be considered as it would relieve aortocaval compression. ${ }^{[8]}$

ICU Management:

Critically ill obstetric patients are managed by a multidisciplinary approach with active involvement of intensivists, obstetricians, nurses, paediatricians, physiotherapists and pharmacists. Maternal haemodynamic optimisation requires application of basic intensive care principles with modifications based on physiological changes during pregnancy.

Monitoring: Parturients should be admitted in the ICU if two organ systems are compromised with a need for ventilatory support. Foetal monitoring must be performed after 24 weeks of gestation to help in determining the adequacy of maternal cardiorespiratory and metabolic parameters. Invasive monitoring may be required in some patients along with minimum mandatory monitoring. The choice of invasive monitoring like central venous pressure (CVP), pulmonary artery (PA) catheter for pulmonary capillary wedge pressure (PCWP), systemic vascular resistance, cardiac output, PA pressure and mixed venous oxygen saturation will depend on the criticality of the ICU patients. ${ }^{[9]}$

Ventilator strategies: Noninvasive positive pressure ventilation (NIPPV) has been shown to benefit a subset of patients with obstructive airway diseases, as well as sleep disordered breathing in pregnancy but can lead to higher risk for aspiration. A low threshold for ETT intubation should be kept in view of high risk for aspiration. Standard protocols for initiation of ventilation in parturients should be in accordance to the physiological changes in the respiratory parameters during pregnancy. Smaller size ETT is required due to airway edema which otherwise increases airway resistance and interferes with successful weaning during prolonged ventilation. When conventional methods of ventilation fail, newer modes 
such as Airway Pressure Release Ventilation (APRV) and high frequency oscillatory ventilation (HFOV) have been tried.[10] Extracorporeal membrane oxygenation (ECMO) should be used early in acute respiratory failure with refractory hypoxemia. Major disadvantage of ECMO is that it exposes the indwelling foetus to extracorporeal circulation and systemic heparinisation and bleeding in the mother.

Drugs: Commonly used antimicrobials such as penicillin, cephalosporin, macrolides and acyclovir fall into category A, whereas aminoglycosides, quinolones, vancomycin and amphotericin fall in category C. Parturients have high incidence of vaginal candidiasis because of increased secretion of sex hormones. Topical azoles should be the first line in the first trimester. There is a raised concern regarding oral fluconazole (category D) being associated with an increased risk of spontaneous abortions and stillbirths in addition to its teratogenicity in high dosage.

Nutrition: Enteral nutrition is considered the optimal method for providing nutrition compared to parenteral nutrition. Aspiration risk can be minimised with antiaspiration prophylaxis (H2 blockers or proton pump inhibitors), prokinetic drugs, using semirecumbent position for administering enteral feeds and routine radiological confirmation of the nasogastric tube position. The basal caloric requirement of a critically ill patient is $25 \mathrm{kcal} / \mathrm{kg} /$ day (ideal body weight) that turns out to be 2200 to $2800 \mathrm{kcal} /$ day for an average sized female. ${ }^{[11]}$ Parturient needs additional $340 \mathrm{kcal} / \mathrm{day}$ and 452 $\mathrm{kcal} /$ day in the second and third trimesters, respectively. The protein requirement is twice that of a nonobstetric patient. Carbohydrates meet up to $70 \%$ caloric requirements while fats meet $30 \%$ caloric requirements. Proteins in diet compensate for negative nitrogen balance and hence are not included in the caloric needs of the critically ill patient. An optimal nutritional solution needs to have additional amounts of zinc, folate and vitamin B12 in the first trimester. The iron content needs to be almost double that of nonobstetric population, corresponding to $4-6 \mathrm{mg}$ /day.

Thromboprophylaxis: Obstetric critically ill patients have four times higher risk of developing deep vein thrombosis compared to other critically ill patients. Patients admitted in ICU must be started on thromboprophylaxis (if there are no other contraindications) as soon as possible. Pharmacological thromboprophylaxis can be achieved with both unfractionated and low molecular weight heparins such as enoxaparin, dalteparin and tinzaparin. However, in case of heparin induced thrombocytopenia (HIT) or severe allergic reactions, a feasible alternative could be provided by argatroban, danaparoid, fondaparinux or lepirudin. ${ }^{[12]}$

Prognostic indicator:

In developed countries mortality rate in obstetric patients ranges from $12-20 \%$ with an admission rate of $3 \%$ of overall ICU admissions while in India mortality rate ranges from $28-60 \%$ and the admission rate to the ICU is $7 \%$. These patients have a better prognosis because delivery will often improve their physiological status.

Different scoring systems are validated for critically ill nonobstetric patients which are either based on physiological variables [Acute Physiology and Chronic Health Evaluation (APACHE) II, Simplified Acute Physiology Score (SAPS) II] or based on organ failure [Sequential Organ Failure Assessment (SOFA), and Multiple Organ Dysfunction Score (MODS)]. Physiology based scores are not very reliable as normal physiological changes of pregnancy make the parameters fall into an abnormal range altering these scores. Sepsis in Obstetrics Score (SOS) that includes parameters like temperature, heart rate, respiratory rate, oxygen saturation, leukocyte count and lactic acid has recently been developed which determines predictability of ICU admission from emergency department (ED). ${ }^{[13]}$ However, organ failure based scores such as MODS have been found to be superior to SOS in mortality prediction in the obstetric patients. ${ }^{[14]}$

Modified Early Warning Scores:

MOEWS(Modified Early Warning Scores) is an important indicator for impending critical illness in the obstetric wards, which includes physiological parameters such as respiratory rate, heart rate, blood pressure, temperature, mental status and urine outputinto the scoring systems. These physiological measurements are incorporated into a scoring system and once a trigger score is reached the patient is referred to the high dependency or intensive care unit. Generally it is observed that Obstetric patients admitted to the ICU have a lower mortality rate than general medical patients. ${ }^{[15]}$

Conclusions:

Optimal management of the critically ill obstetric patient involve early detection and multi disciplinary approach with close collaboration of the critical care team including obstetricians, midwives, anesthesiologists and neonatologists based on knowledge of physiological and pharmacological alterations. Thus management of obstetric complications in a dedicated HDU or obstetric ICU can improve both maternal and foetal outcome, especially in less resourced settings.

Reference:

1. Pollock W, Rose L, Dennis CL. Pregnant and postpartum admissions to the intensive care unit: A systematic review. Intensive Care Med 2010;36:1465-74.

2. Plaat F, Wray S. Role of the anaesthetist in obstetric critical care. Best Pract Res Clin Obstet Gynaecol 2008;22(5):917-35

3. Zeeman GG, Wendel GD Jr, Cunningham FG. A blueprint for obstetric critical care. Am J Obstet Gynecol 2003;188:532-6.

4. Kaur M, Singh PM, Trikha A. Management of critically ill Obstetric patients: A review. J Obstet Anesth Crit Care 2017;7:3-12.

5. Butwick AJ, Goodnough LT. Transfusion and coagulation management in major obstetric hemorrhage. CurrOpinAnaesthesiol $2015 ; 28: 275-84$

6. Galvão A, Braga AC, Gonçalves DR, Guimarães JM, Braga J. Sepsis during pregnancy or the postpartum period. J ObstetGynaecol J InstObstetGynaecol 2016;1-9.

7. Hu H, Pasca I. Management of Complex Cardiac Issues in the Pregnant Patient. Crit Care Clin 2016;32:97-107.

8. Lavonas EJ, Drennan IR, Gabrielli A, Heffner AC, Hoyte CO, Orkin AM, et al. Part 10: Special Circumstances of Resuscitation: 2015 American Heart Association Guidelines Update for Cardiopulmonary Resuscitation and Emergency Cardiovascular Care. 
Circulation 2015;132(18 Suppl 2):S501-18.

9. Ivanov RI, Allen J, Sandham JD, Calvin JE. Pulmonary artery catheterization: A narrative and systematic critique of randomized controlled trials and recommendations for the future. New Horiz 1997;5:268-76.

10. Hirani A, Marik PE, Plante LA. Airway pressure-release ventilation in pregnant patients with acute respiratory distress syndrome: A novel strategy. Respir Care 2009;54:1405-8.

11. Cox JT, Phelan ST. Nutrition during pregnancy. ObstetGynecolClin North Am 2008;35:369-383.

12. Palmerola K, D’Alton M, Brock C, Friedman A. A comparison of recommendations for pharmacologic thromboembolism prophylaxis after caesarean delivery from three major guidelines. BJOG Int J ObstetGynaecol 2016;123:2157-62.

13. Albright CM, Ali TN, Lopes V, Rouse DJ, Anderson BL. The Sepsis in Obstetrics Score: A model to identify risk of morbidity from sepsis in pregnancy. Am J ObstetGynecol 2014;211:39.e1-8.

14. Aarvold ABR, Ryan HM, Magee LA, von Dadelszen P, Fjell C, Walley KR. Multiple Organ Dysfunction Score Is Superior to the Obstetric-Specific Sepsis in Obstetrics Score in Predicting Mortality in Septic Obstetric Patients. Crit Care Med 2017;45:e49-57.

15. Gupta S. Clinical Charecteristics and outcome of obstetric patients requiring ICU admission. Year book of AnaesthesiologyVol 3 Ed. Chandra U, Basu SM. Ch 3,pp 27-36. 